\title{
NON-COMPLETENESS OF THE ARAKELOV-INDUCED METRIC ON MODULI SPACE OF CURVES
}

\author{
J. JORGENSON AND J. KRAMER
}

\section{INTRODUCTION}

1.1. Weil-Petersson metric on the moduli space of curves. The classical theory of the moduli space of compact Riemann surfaces of a fixed genus $g>1$ includes the investigation of the Weil-Petersson metric. Briefly, consider any compact Riemann surface $X$ of genus $g>1$, which represents a point in the moduli space $\mathcal{M}_{g}$ of such objects. Then, the tangent space to $X$ in $\mathcal{M}_{g}$ is naturally dual to $H^{0}\left(X, \Omega_{X}^{2}\right)$, the $\mathbb{C}$-vector space of holomorphic quadratic differentials on $X$. Let $\mu$ be a smooth, positive $(1,1)$-form on $X$ corresponding to a smooth metric on $X$. For any $\omega, \eta \in H^{0}\left(X, \Omega_{X}^{2}\right)$, the classical Weil-Petersson inner product is defined by

$$
\langle\omega, \eta\rangle=\frac{i}{2} \int_{X}(\omega \wedge \bar{\eta}) \cdot \mu^{-1} .
$$

By duality, a metric on the tangent space of $\mathcal{M}_{g}$ at the point represented by $X$ is induced by (1). When taking $\mu$ to be the $(1,1)$-form $\mu_{\text {hyp }}$ associated to the hyperbolic metric on $X$, one obtains the classically studied Weil-Petersson metric on $\mathcal{M}_{g}$; basic questions such as the completeness, curvature, and Kählerian aspects of the Weil-Petersson metric on $\mathcal{M}_{g}$ are well-known (see [5], e.g., and references therein).

1.2. Arakelov metrics on Riemann surfaces. Much of the existing literature focuses on the Weil-Petersson metric on $\mathcal{M}_{g}$, which is induced from the family of hyperbolic metrics on the underlying universal family of Riemann surfaces. Indeed, from the uniformization theorem, the family of hyperbolic metrics is a natural candidate from which one can form the associated Weil-Petersson metric on $\mathcal{M}_{g}$. However, other points of view yield

The first author acknowledges support from grants from the NSF and PSC-CUNY. The second author thanks the Centre de Recerca Matemàtica (CRM) in Barcelona for its support and hospitality. 
different families of metrics, and each family of metrics has an associated Weil-Petersson metric on $\mathcal{M}_{g}$.

The family of canonical metrics $\mu_{\text {can }}$ arises from classical algebraic geometry when embedding $X$ into its Jacobian variety via the Abel-Jacobi map, and the articles [3], 4] initiated the study of the corresponding Weil-Petersson metric on $\mathcal{M}_{g}$. From arithmetic algebraic geometry, one has the family of Arakelov metrics $\mu_{\mathrm{Ar}}$, as defined in [1], which provides yet another candidate of metrics from which one can form a Weil-Petersson metric on $\mathcal{M}_{g}$. In [3] and 4], the authors posed the problem of developing a means by which one can study the resulting Arakelov-induced metric on $\mathcal{M}_{g}$. Specifically, it is asked if the Arakelov-induced metric on the moduli space $\mathcal{M}_{g}$ is complete or not, which in the case of the classical Weil-Petersson metric was first answered in [13] and [11].

1.3. The main result. The purpose of the present paper is to answer the problem posed in 3 and 4 concerning the Arakelov-induced metric on $\mathcal{M}_{g}$. The main result we prove in this article is the following. Let $X_{t}(t \in \mathbb{C},|t|<1)$ be a family of compact Riemann surfaces of fixed genus $g>1$ degenerating at $t=0$, and, hence, approaching the DeligneMumford boundary of the moduli space $\mathcal{M}_{g}$; let $\mu_{\mathrm{Ar}, t}$, resp. $\mu_{\mathrm{hyp}, t}$ be the corresponding family of $(1,1)$-forms associated to the Arakelov, resp. hyperbolic metric. Then, we have

$$
\lim _{t \rightarrow 0}\left(\sup _{z \in X_{t}}\left(\frac{\mu_{\mathrm{Ar}, t}(z)}{\mu_{\mathrm{hyp}, t}(z)}\right)\right)=0 .
$$

As a corollary of this result, we prove that the Arakelov-induced metric on $\mathcal{M}_{g}$ is not complete.

1.4. Outline of the paper. In section 2 , we establish notation and recall various known results, most importantly for our purposes here is section 2.4, which expresses $\phi_{\mathrm{Ar}}=\log \left(\mu_{\mathrm{Ar}} / \mu_{\mathrm{hyp}}\right)$, the conformal factor relating the Arakelov metric to the hyperbolic metric, in terms of the hyperbolic heat kernel, a result which has been proved in [9]. In section 3, we study each quantity in the formula for $\phi_{\mathrm{Ar}}$. Ultimately, we bound $\phi_{\mathrm{Ar}}$ using known results for the hyperbolic heat kernel on a degenerating family of compact Riemann surfaces of genus $g>1$, most notably from [10. Finally, in section 4 , we show how our main theorem answers the question of completeness of the Arakelov-induced metric on $\mathcal{M}_{g}$. 


\section{BACKGROUND MATERIAL}

2.1. Hyperbolic and canonical metrics. Let $X$ be a compact Riemann surface of genus $g>1$. By the uniformization theorem, we can identify $X$ complex analytically with the quotient space $\Gamma \backslash \mathbb{H}$, where $\Gamma$ is a Fuchsian subgroup of the first kind of $\mathrm{PSL}_{2}(\mathbb{R})$ acting by fractional linear transformations on the upper half-plane $\mathbb{H}=\{z \in \mathbb{C} \mid \operatorname{Im}(z)>0\}$. In a slight abuse of notation, we will throughout this article identify $X$ with a fundamental domain (say, a Ford domain, bounded by geodesic paths) and identify points on $X$ with their pre-images in $\mathbb{H}$. Let $\mu$ denote a smooth, positive $(1,1)$-form on $X$ corresponding to a smooth metric on $X$. We write $\operatorname{vol}_{\mu}(X)$ for the volume of $X$ with respect to $\mu$. In particular, we let $\mu_{\text {hyp }}$ denote the $(1,1)$-form corresponding to the hyperbolic metric on $X$, which is compatible with the complex structure of $X$, and has constant negative curvature equal to minus one. As a shorthand, we write $v_{\text {hyp }}$ for the hyperbolic volume $\operatorname{vol}_{\mu_{\text {hyp }}}(X)$; we recall that $v_{\text {hyp }}=4 \pi(g-1)$.

Let $H^{0}\left(X, \Omega_{X}^{1}\right)$ denote the $\mathbb{C}$-vector space of holomorphic 1-forms equipped with the Petersson inner product given by

$$
\langle\omega, \eta\rangle=\frac{i}{2} \int_{X} \omega \wedge \bar{\eta} \quad\left(\omega, \eta \in H^{0}\left(X, \Omega_{X}^{1}\right)\right) .
$$

By choosing an orthonormal basis $\left\{\omega_{1}, \ldots, \omega_{g}\right\}$ of $H^{0}\left(X, \Omega_{X}^{1}\right)$ with respect to the Petersson inner product, the canonical metric of $X$ has the associated $(1,1)$-form

$$
\mu_{\mathrm{can}}=\frac{1}{g} \cdot \frac{i}{2} \sum_{j=1}^{g} \omega_{j} \wedge \bar{\omega}_{j}
$$

We note that the canonical metric measures the volume of $X$ to be one, and, furthermore, $\mu_{\text {can }}$ is independent of the choice of orthonormal basis of $H^{0}\left(X, \Omega_{X}^{1}\right)$.

We write $\Delta_{\text {hyp }}$ for the hyperbolic Laplacian on $X$, which acts on smooth functions, and we let $\left\{\phi_{n}\right\}_{n=0}^{\infty}$ denote an orthonormal basis of eigenfunctions of $\Delta_{\text {hyp }}$ on $X$ with corresponding eigenvalues

$$
0=\lambda_{0}<\lambda_{1} \leq \lambda_{2} \leq \ldots,
$$

i.e.,

$$
\Delta_{\text {hyp }} \phi_{n}=\lambda_{n} \phi_{n} \quad(n=0,1,2, \ldots)
$$

2.2. Green's functions and Arakelov metrics. We denote the Green's function associated to the metric $\mu$ by $g_{\mu}$. It is a function on $X \times X$ 
characterized by the two properties

$$
\begin{gathered}
\mathrm{d}_{z} \mathrm{~d}_{z}^{c} g_{\mu}(z, w)+\delta_{w}(z)=\frac{\mu(z)}{\operatorname{vol}_{\mu}(X)}, \\
\int_{X} g_{\mu}(z, w) \mu(z)=0 \quad(w \in X) .
\end{gathered}
$$

Assuming that $z, w$ are points on $X$, which are sufficiently close, our convention for the Green's function is such that the sum $g_{\mu}(z, w)+\log |z-w|^{2}$ is bounded as $w$ approaches $z$. By means of the function $G_{\mu}=\exp \left(g_{\mu}\right)$, we now define the residual metric $\|\cdot\|_{\mu \text {,res }}$ on the canonical line bundle $\Omega_{X}^{1}$ associated to $\mu$ by

$$
\|\mathrm{d} z\|_{\mu, \mathrm{res}}^{2}=\lim _{w \rightarrow z}\left(G_{\mu}(z, w) \cdot|z-w|^{2}\right) .
$$

The (1,1)-form associated to the residual metric is then given by

$$
\mu_{\mathrm{res}}(z)=\frac{i}{2} \cdot \frac{\mathrm{d} z \wedge \mathrm{d} \bar{z}}{\|\mathrm{~d} z\|_{\mu, \text { res }}^{2}} .
$$

In particular, following [1, we define the Arakelov metric form $\mu_{\mathrm{Ar}}$ to be the (1,1)-form associated to the residual metric of the canonical metric; the corresponding metric on $\Omega_{X}^{1}$ is denoted by $\|\cdot\|_{\mathrm{Ar}}$. The conformal factor $\phi_{\mathrm{Ar}}$ is the $C^{\infty}$-function on $X$ defined by the equation

$$
\mu_{\mathrm{Ar}}=e^{\phi_{\mathrm{Ar}}} \mu_{\mathrm{hyp}} \text {. }
$$

2.3. Heat kernels and heat traces. The heat kernel $K_{\mathbb{H}}(t ; z, w)$ on $\mathbb{H}$ $\left(t \in \mathbb{R}_{>0} ; z, w \in \mathbb{H}\right)$ is given by the formula

$$
K_{\mathbb{H}}(t ; z, w)=K_{\mathbb{H}}(t ; \rho)=\frac{\sqrt{2} e^{-t / 4}}{(4 \pi t)^{3 / 2}} \int_{\rho}^{\infty} \frac{r e^{-r^{2} / 4 t}}{\sqrt{\cosh (r)-\cosh (\rho)}} \mathrm{d} r
$$

where $\rho=d_{\mathbb{H}}(z, w)$ denotes the hyperbolic distance between $z$ and $w$. Evidentially, $K_{\mathbb{H}}(t ; z, w)>0$ for $t>0$ and $z, w \in \mathbb{H}$. The heat kernel $K_{\text {hyp }}(t ; z, w)$ associated to $X\left(t \in \mathbb{R}_{>0} ; z, w \in X\right)$ is defined by averaging over the elements of $\Gamma$, namely

$$
K_{\text {hyp }}(t ; z, w)=\sum_{\gamma \in \Gamma} K_{\mathbb{H}}(t ; z, \gamma w) .
$$

For $t>0$ and $z \in X$, we let

$$
H K_{\text {hyp }}(t ; z)=K_{\text {hyp }}(t ; z, z)-K_{\mathbb{H}}(t ; 0)=\sum_{\substack{\gamma \in \Gamma \\ \gamma \neq \text { id }}} K_{\mathbb{H}}(t ; z, \gamma z) .
$$


Let us define the function

$$
F(z)=\int_{0}^{\infty}\left(H K_{\text {hyp }}(t ; z)-\frac{1}{v_{\text {hyp }}}\right) \mathrm{d} t .
$$

From standard results regarding the asymptotic behavior of the heat kernel for $t \rightarrow 0$ and $t \rightarrow \infty$, the integral defining $F$ converges for all $z \in X$. Since $X$ is compact, the heat kernel admits the spectral expansion

$$
K_{\mathrm{hyp}}(t ; z, w)=\sum_{n=0}^{\infty} \phi_{n}(z) \phi_{n}(w) e^{-\lambda_{n} t}
$$

which can be shown to converge uniformly and absolutely on $X$. The hyperbolic heat trace $H \operatorname{Tr} K_{\text {hyp }}(t)\left(t \in \mathbb{R}_{>0}\right)$ is now given by

$$
H \operatorname{Tr} K_{\mathrm{hyp}}(t)=\int_{X} H K_{\mathrm{hyp}}(t ; z) \mu_{\mathrm{hyp}}(z) .
$$

The logarithmic derivative of the Selberg zeta function $Z_{\mathrm{Sel}}(s)$ associated to $X$ is defined by the Gauss transform of the hyperbolic heat trace, namely

$$
\frac{Z_{\text {Sel }}^{\prime}}{Z_{\text {Sel }}}(s)=(2 s-1) \int_{0}^{\infty} H \operatorname{Tr} K_{\text {hyp }}(t) e^{-s(s-1) t} \mathrm{~d} t,
$$

which converges for $\operatorname{Re}(s(s-1))>0$ and, when combined with the spectral expansion of the heat kernel, can be used to obtain the analytic continuation of $Z_{\mathrm{Sel}}(s)$. Of particular interest is the constant

$$
c_{\text {Sel }}=\lim _{s \rightarrow 1}\left(\frac{Z_{\text {Sel }}^{\prime}}{Z_{\text {Sel }}}(s)-\frac{1}{s-1}\right),
$$

which is analogous to the classical $\eta$-function as realized via the Kronecker limit formula. From [7, Lemma 4.2, we recall that one has the explicit formula for $c_{\text {Sel }}$ in terms of the hyperbolic heat trace, namely

$$
c_{\text {Sel }}=1+\int_{0}^{\infty}\left(H \operatorname{Tr} K_{\text {hyp }}(t)-1\right) \mathrm{d} t=\int_{0}^{\infty}\left(H \operatorname{Tr} K_{\text {hyp }}(t)-1+e^{-t}\right) \mathrm{d} t .
$$

2.4. Arakelov invariants using hyperbolic geometry. Numerous authors have studied the analytic invariants from the Arakelov theory of algebraic curves with the goal of obtaining expressions for the various quantities, including: the canonical metric, the canonical Green's function, the Arakelov metric, and Faltings's delta function. Beginning with [6] and [12], all authors relied on algebraic geometry and used the Riemann theta function as the fundamental object from which various identities were derived. 
In [9], new relations were derived relating the analytic invariants of the Arakelov theory of curves to the hyperbolic heat kernel. For the present paper, we will work with the following result, first proved in 9 (see also [8]). If $X$ is any compact Riemann surface of genus $g>1$, then, in the notation of the previous section, we have

$\phi_{\mathrm{Ar}}(z)=-4 \pi\left(1-\frac{1}{g}\right) F(z)-\frac{\pi}{g^{2}} \int_{X} F(z) \Delta_{\mathrm{hyp}} F(z) \mu_{\mathrm{hyp}}(z)-\frac{c_{\mathrm{Sel}}-1}{g(g-1)}-\log (4)$.

The proof of (3) follows from more fundamental formulas which relate $\mu_{\text {can }}$ to $\mu_{\text {hyp }}$ and $g_{\text {can }}$ to $g_{\text {hyp }}$; these proofs are given in [8], [9], and will not be repeated here. Also, we note that other results from [9] yield explicit expressions for the canonical Green's function, Faltings's delta function, and the canonical metric form in terms of the hyperbolic heat kernel.

2.5. Degenerating families of Riemann surfaces. By a degenerating family of compact Riemann surfaces $X_{t}(t \in D=\{t \in \mathbb{C}|| t \mid<1\})$, we mean a holomorphic map of $D$ into the stably (Deligne-Mumford) compactified moduli space such that the restriction of this map to the punctured disc $D \backslash\{0\}$ is a holomorphic map into the moduli space $\mathcal{M}_{g}$. The fiber over the origin $t=0$ of the embedded disc is a noded algebraic curve. We refer the reader to [2] for an explicit construction of degenerating families of Riemann surfaces. Although the discussion in 2] focuses on degenerating families with a single developing node, the methodology immediately extends to the general setting of an arbitrary number of developing nodes. If each surface in the family $X_{t}$ is given its unique hyperbolic metric, then we call the family a degenerating family of compact hyperbolic Riemann surfaces. The family can be parameterized either by the complex parameter $t \in D$ or the vector $\ell$ of lengths of pinching hyperbolic geodesics. We refer the reader to [6], [10, and references therein for further discussion.

\section{Asymptotic Behavior of the Arakelov Conformal factor}

In this section we estimate the terms in (3), which involve the hyperbolic heat kernel.

3.1. Lemma. For any compact Riemann surface $X$ of genus $g>1$, we have the bound

$$
\int_{X} F(z) \Delta_{\mathrm{hyp}} F(z) \mu_{\mathrm{hyp}}(z) \geq 0
$$


Proof. Since $X$ is compact, we can expand $F$ in terms of the orthonormal basis of eigenfunctions $\left\{\phi_{n}\right\}_{n=0}^{\infty}$ of $\Delta_{\text {hyp }}$ with the non-negative eigenvalues $\left\{\lambda_{n}\right\}_{n=0}^{\infty}$, i.e.,

$$
F(z)=\sum_{n=0}^{\infty} a_{n} \phi_{n}(z)
$$

from which we derive

$$
\Delta_{\text {hyp }} F(z)=\sum_{n=1}^{\infty} \lambda_{n} a_{n} \phi_{n}(z),
$$

taking into account that $\lambda_{X, 0}=0$. Therefore, we have the equality

$$
\int_{X} F(z) \Delta_{\mathrm{hyp}} F(z) \mu_{\mathrm{hyp}}(z)=\sum_{n=1}^{\infty} \lambda_{n} a_{n}^{2}
$$

from which the assertion in the statement of the lemma follows since $\lambda_{n} \geq 0$ for all $n$.

3.2. Lemma. For any compact Riemann surface $X$ of genus $g>1$, we have the bound

$$
F(z) \geq-\frac{1}{v_{\text {hyp }}}-\int_{1}^{\infty} K_{\mathbb{H}}(t ; 0) \mathrm{d} t .
$$

Proof. If $t \geq 1$, we have the bound

$$
\begin{aligned}
& H K_{\text {hyp }}(t ; z)-\frac{1}{v_{\text {hyp }}}=K_{\text {hyp }}(t ; z, z)-\frac{1}{v_{\text {hyp }}}-K_{\mathbb{H}}(t ; 0)= \\
& \sum_{n=1}^{\infty} \phi_{n}(z)^{2} e^{-\lambda_{n} t}-K_{\mathbb{H}}(t ; 0) \geq-K_{\mathbb{H}}(t ; 0),
\end{aligned}
$$

which simply uses the positivity of the sum of squares of eigenfunctions. If $0<t \leq 1$, we trivially have

$$
H K_{\text {hyp }}(t ; z)-\frac{1}{v_{\text {hyp }}} \geq-\frac{1}{v_{\text {hyp }}},
$$

which, using the positivity of $K_{\mathbb{H}}(t ; z, w)$, follows immediately from the positivity of $H K_{\text {hyp }}(t ; z)$. With these bounds, the result immediately follows from the integral (2), which defines $F(z)$. 
3.3. Corollary. For any compact Riemann surface $X$ of genus $g>1$, we have the bound

$\log \left(\frac{\mu_{\mathrm{Ar}}(z)}{\mu_{\mathrm{hyp}}(z)}\right)=\phi_{\mathrm{Ar}}(z) \leq 4 \pi\left(1-\frac{1}{g}\right) \int_{1}^{\infty} K_{\mathbb{H}}(t ; 0) \mathrm{d} t-\frac{c_{\text {Sel }}}{g(g-1)}+\frac{1}{g-1}-\log (4)$.

Proof. By combining the bounds in Lemma 3.1 and Lemma 3.2 with (3), we immediately arrive at the inequality

$$
\phi_{\mathrm{Ar}}(z) \leq 4 \pi\left(1-\frac{1}{g}\right)\left(\frac{1}{v_{\text {hyp }}}+\int_{1}^{\infty} K_{\mathbb{H}}(t ; 0) \mathrm{d} t\right)-\frac{c_{\mathrm{Sel}}-1}{g(g-1)}-\log (4) .
$$

The bound follows by combining the constants, namely by showing that

$$
4 \pi\left(1-\frac{1}{g}\right) \frac{1}{v_{\text {hyp }}}+\frac{1}{g(g-1)}=\frac{1}{g-1},
$$

which comes from $v_{\text {hyp }}=4 \pi(g-1)$.

3.4. Lemma. Let $X_{t}$ be a degenerating family of compact hyperbolic Riemann surfaces of genus $g>1$. Writing $c_{S e l, t}$ for the constants $c_{S e l}$ associated to $X_{t}$, we have

$$
\lim _{t \rightarrow 0} c_{\mathrm{Sel}, t}=\infty
$$

Proof. The lemma is a direct consequence of [10], Theorem 4.5, p. 657, which we will briefly describe for the sake of completeness.

Writing $X_{t}=\Gamma_{t} \backslash \mathbb{H}$, and given $\varepsilon_{\text {geo }}>0$, resp. $\varepsilon_{\text {ev }}>0$, we let

$$
\begin{aligned}
& \mathcal{G}_{\text {geo }}=\left\{\gamma \in \Gamma_{t} \mid \gamma \neq \mathrm{id}, \text { primitive, non-conjugate, } \ell_{\gamma}<\varepsilon_{\text {geo }}\right\}, \text { resp. } \\
& \mathcal{G}_{\text {ev }}=\left\{\lambda_{n, t} \mid \lambda_{n, t} \text { eigenvalues of } \Delta_{\text {hyp }} \text { on } X_{t}, 0<\lambda_{n, t}<\varepsilon_{\text {ev }}\right\}
\end{aligned}
$$

here $\ell_{\gamma}$ denotes the hyperbolic length of the primitive geodesic associated to $\gamma \in \Gamma_{t}$. Choosing $\varepsilon_{\text {geo }}$ sufficiently small, and $\varepsilon_{\mathrm{ev}}<1 / 4$, the above cited theorem of [10] gives the following asymptotic formula for the quantity $c_{\mathrm{Sel}, t}$, as $t$ approaches zero:

$$
c_{\mathrm{Sel}, t}=\sum_{n=1}^{\infty} \sum_{\gamma \in \mathcal{G}_{\mathrm{geo}}} \frac{\ell_{\gamma}}{e^{n \ell_{\gamma}}-1}+\sum_{\lambda_{n, t} \in \mathcal{G}_{\mathrm{ev}}} \frac{1}{\lambda_{n, t}}+\mathrm{O}_{\varepsilon_{\mathrm{geo}}, \varepsilon_{\mathrm{ev}}}(1) .
$$

We recall from [10] (and the references therein) that as $t$ approaches zero,

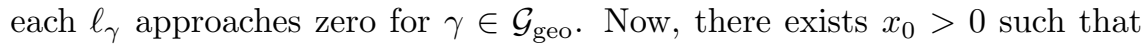


for $0<x<x_{0}$, the inequality $e^{x}-1 \leq 2 x$ holds. With this in mind, we obtain the bound

$$
c_{\text {Sel }, t} \geq \sum_{\gamma \in \mathcal{G}_{\text {geo }}} \sum_{n<x_{0} / \ell_{\gamma}} \frac{1}{2 n}+\sum_{\lambda_{n, t} \in \mathcal{G}_{\mathrm{ev}}} \frac{1}{\lambda_{n, t}}+\mathrm{O}_{\varepsilon_{\text {geo }}, \varepsilon_{\mathrm{ev}}}(1) .
$$

Using the positivity of the eigenvalues and the divergence of the harmonic series, the proof of the lemma is now complete.

3.5. Theorem. Let $X_{t}$ be a degenerating family of compact hyperbolic Riemann surfaces of genus $g>1$. Writing $\mu_{\text {hyp }, t}$, resp. $\mu_{A r, t}$ for the $(1,1)$ forms $\mu_{\text {hyp }}$, resp. $\mu_{A r}$ associated to $X_{t}$, we have

$$
\lim _{t \rightarrow 0}\left(\sup _{z \in X_{t}}\left(\frac{\mu_{\mathrm{Ar}, t}(z)}{\mu_{\mathrm{hyp}, t}(z)}\right)\right)=0 .
$$

Proof. Combine Corollary 3.3 and Lemma 3.4 .

3.6. Remark. The analysis given in 6 and 12 do not seem sufficient to prove Theorem 3.5. More specifically, although the asymptotic behavior of $\mu_{\mathrm{Ar}, t}$ is established for points which are bounded away from the developing nodes, the results in [6] and [12] do not establish bounds for $\mu_{\mathrm{Ar}, t} / \mu_{\mathrm{hyp}, t}$ in neighborhoods of the developing nodes. As a consequence, it seems that Theorem 3.5 does not follow easily, if at all, from the results in [6] and 12 .

\section{The Arakelov-induced metric on moduli spaCe}

We show in this section how Theorem 3.5 can be used to study the metric on the moduli space $\mathcal{M}_{g}$ near the Deligne-Mumford boundary.

4.1. Arakelov-induced metric on the moduli space. As before, let $X$ denote a compact Riemann surface of genus $g>1$. Then, the $\mathbb{C}$-vector space $H^{0}\left(X, \Omega_{X}^{2}\right)$ of holomorphic quadratic differentials on $X$ has dimension $(3 g-$ $3)$. Its dual space $H^{0}\left(X, \Omega_{X}^{2}\right)^{\vee}$ consists of harmonic Beltrami differentials; it represents the tangent space to the point corresponding to $X$ in the appropriate Teichmüller space $\mathcal{T}_{g}$. The Arakelov-induced inner product $\langle\cdot, \cdot\rangle$ on $H^{0}\left(X, \Omega_{X}^{2}\right)^{\vee}$ is defined by

$$
\langle\mu, \nu\rangle=\frac{i}{2} \int_{X}(\mu \wedge \bar{\nu}) \cdot \mu_{\mathrm{Ar}} \quad\left(\mu, \nu \in H^{0}\left(X, \Omega_{X}^{2}\right)^{\vee}\right) .
$$

To be precise, the Arakelov metric $\mu_{\mathrm{Ar}}$ on $X$ induces a metric on the Teichmüller space $\mathcal{T}_{g}$ of marked Riemann surfaces of genus $g>1$; we use the term "marked" to denote that a canonical basis of the homology group $H_{1}(X, \mathbb{Z})$ has been chosen. However, the Arakelov metric is independent of 
the marking chosen, which is evident since the canonical metric, hence the canonical Green's function, is independent of any marking chosen. Consequently, the Arakelov-induced metric on the Teichmüller space descends to a metric on the moduli space $\mathcal{M}_{g}$.

4.2. Theorem. The Arakelov-induced metric on $\mathcal{M}_{g}$ is not complete.

Proof. Consider a degenerating family $X_{t}$ of compact Riemann surfaces of genus $g>1$, whose limiting surface $X_{0}$ corresponds to a reducible, uninoded algebraic curve, i.e., in the language of hyperbolic geometry, the family $X_{t}$ has a single pinching geodesic which separates each Riemann surface into two components. Let $L_{\mathcal{M}_{g} \text {,hyp }}\left(X_{t}\right)$, resp. $L_{\mathcal{M}_{g}, \text { Ar }}\left(X_{t}\right)$ denote the length of the path in $\mathcal{M}_{g}$ associated to the family $X_{t}$ measured using the hyperbolicinduced, resp. Arakelov-induced metric on $\mathcal{M}_{g}$. In [11, it is shown that $L_{\mathcal{M}_{g} \text {,hyp }}\left(X_{t}\right)$ is finite, i.e., the Weil-Petersson metric associated to the family of hyperbolic metrics is not complete. Now, for a fixed point in $\mathcal{M}_{g}$, corresponding to the Riemann surface $X$, we can write

$$
\langle\mu, \nu\rangle=\frac{i}{2} \int_{X}(\mu \wedge \bar{\nu}) \cdot \mu_{\mathrm{Ar}}=\frac{i}{2} \int_{X}(\mu \wedge \bar{\nu}) \cdot e^{\phi_{\mathrm{Ar}}} \mu_{\mathrm{hyp}} \cdot
$$

Therefore, by Theorem 3.5, there is a constant $C>0$ such that $L_{\mathcal{M}_{g}, \text { Ar }}\left(X_{t}\right) \leq C \cdot L_{\mathcal{M}_{g} \text {,hyp }}\left(X_{t}\right)$. Since $L_{\mathcal{M}_{g} \text {,hyp }}\left(X_{t}\right)$ is finite, so is $L_{\mathcal{M}_{g}, \operatorname{Ar}}\left(X_{t}\right)$, which proves the theorem.

4.3. Remark. As Theorem 4.2 demonstrates, the identity (3) allows one to study the Arakelov-induced metric on $\mathcal{M}_{g}$ going beyond results obtainable through other techniques, such as in [3] and [4. Going further, one now has the opportunity to study other properties of the Arakelov-induced metric on $\mathcal{M}_{g}$ by following the methodology of Theorem 4.2: Utilize the corresponding result in the classical Weil-Petersson (hyperbolic-induced) setting, together with properties of the hyperbolic heat kernel. We leave for elsewhere the problem of systematically studying the Arakelov-induced metric on $\mathcal{M}_{g}$, as first posed in [3] and [4], using the identity (3).

\section{REFERENCES}

[1] G. Faltings, Calculus on arithmetic surfaces, Ann. of Math. 119 (1984), 387-424.

[2] J. Fay, Theta functions on Riemann surfaces, Lecture Notes in Math. 352, SpringerVerlag, Berlin-Heidelberg-New York, 1973.

[3] L. Habermann, J. Jost, Riemannian metrics on Teichmüller space, Manuscripta Math. 89 (1996), 281-306.

[4] L. Habermann, J. Jost, Metrics on Riemann surfaces and the geometry of moduli spaces, Sympos. Math. XXXVIII, Cambridge Univ. Press, Cambridge (1998), 53-70. 
[5] Y. Imayoshi, M. Taniguchi, An introduction to Teichmüller spaces, Springer-Verlag, Berlin, 1992.

[6] J. Jorgenson, Asymptotic behavior of Faltings's delta function, Duke Math J. 61 (1990), 221-254

[7] J. Jorgenson, J. Kramer, Bounds on special values of Selberg's zeta functions for Riemann surfaces, J. reine angew. Math 541 (2001), 1-28.

[8] J. Jorgenson, J. Kramer, Bounds on Faltings's delta function through covers, submitted.

[9] J. Jorgenson, J. Kramer, Expressing Arakelov invariants using hyperbolic heat kernels, to appear in: The Ubiquitous Heat Kernel, Contemp. Math. AMS, 2006.

[10] J. Jorgenson, R. Lundelius, A regularized heat trace for hyperbolic Riemann surfaces of finite volume, Comment. Math. Helv. 72 (1997), 636-659.

[11] H. Masur, The extension of the Weil-Petersson metric to the boundary of Teichmüller space, Duke Math. J. 43 (1976), 623-635.

[12] R. Wentworth, The asymptotics of the Arakelov-Green's function and Faltings' delta invariant, Commun. Math. Phys. 137 (1991), 427-459.

[13] S. Wolpert, Non-completeness of the Weil-Petersson metric for Teichmüller space, Pacific J. Math 61 (1975), 573-577.

Jay Jorgenson

Department of Mathematics

The City College of New York

Convent Avenue at 138th Street

New York, NY 10031 U.S.A.

e-mail: jjorgenson@mindspring.com

Jürg Kramer

Institut für Mathematik

Humboldt-Universität zu Berlin

Unter den Linden 6

D-10099 Berlin

Germany

e-mail: kramer@math.hu-berlin.de 